\title{
Kinetics of random sequential adsorption of rectangles and line segments
}

\author{
R. Dennis Vigi|a) and Robert M. Ziff \\ Department of Chemical Engineering, The University of Michigan, Ann Arbor, Michigan 48109
}

(Received 28 June 1990; accepted 17 August 1990)

\begin{abstract}
Random sequential adsorption of nonoverlapping rectangles of arbitrary orientation onto a continuous plane was investigated by computer simulation. It is shown that the approach to the jamming limit of rectangles with various aspect ratios obeys the scaling law $\theta_{J}-\theta(\tau) \sim \tau^{-1 / 3}$. Furthermore, the adsorption kinetics of rectangles approach those of line segments as the rectangle aspect ratio becomes large.
\end{abstract}

\section{INTRODUCTION}

Random sequential adsorption (RSA) is an irreversible process in which objects are placed, one at a time, with random position and orientation, subject to the condition that they do not overlap previously adsorbed objects. Eventually, no more objects can be adsorbed and the so-called "jamming limit" is reached.

This problem was first studied in one dimension and complete analytic solutions have been derived for the kinetics of adsorption for both discrete and continuous cases. ${ }^{1-5}$ Recently, there has been much interest in random sequential adsorption in two and higher spatial dimensions, but no analytic solutions are known for the adsorption kinetics. $^{6-9}$ Feder $^{10}$ investigated the kinetics of RSA of disks on a plane (through the use of computer simulations) and he postulated the following scaling law for the long-time behavior of the surface coverage fraction $\theta$ :

$$
\theta_{J}-\theta(\tau) \sim \tau^{-p}, \quad \tau \rightarrow \infty,
$$

where $\tau$ is the cumulative number of attempts to adsorb objects (both successful and not) and $\theta_{J}$ is the surface coverage at the jammed state. Feder observed $p \approx 1 / 2$ and later Pomeau ${ }^{11}$ and Swendsen ${ }^{12}$ constructed arguments based upon geometrical probability that confirm this result. Furthermore, Swendsen ${ }^{12}$ has argued that it may be generalized for arbitrary shapes, provided that objects are adsorbed with random orientations. Note that other models of RSA do not necessarily obey Eq. (1). For example, Swendsen ${ }^{12}$ argues that the kinetics of RSA of aligned squares obeys

$$
\theta_{J}-\theta(\tau) \sim \frac{\ln \tau}{\tau}, \quad \tau \rightarrow \infty
$$

Recent computer simulations of the RSA of aligned squares give close agreement with this prediction. ${ }^{13}$

Talbot, Tarjus, and Schaaf ${ }^{8}$ have argued that the longtime kinetics of RSA of noncircular objects obeys the scaling law (1) with $p=1 / 3$ rather than $1 / 2$, due to the orientational restrictions that are not present for the case of circles. That is, as the jamming limit is approached, the success of a given adsorption attempt of noncircular objects depends both on the position on the surface at which the object lands and the angular orientation of the object.
They have performed computer simulations of the RSA of ellipses with aspect ratios between 1 and 5 and have measured values of $p$ in the range $0.2-0.5$. Although their simulations are consistent with the prediction $p=1 / 3$, they are less than conclusive. At the same time that Talbot $e t$ al. presented these findings, the present authors described a study of RSA of unoriented rectangles. ${ }^{9}$ While the main interest in that work was the determination of $\theta_{J}$ as a function of aspect ratio $\alpha$, we also carried out a preliminary study of the time dependence and found that the data is more consistent with the prediction of $p=1 / 3$ than with $p=1 / 2$.

In this paper we describe a more thorough investigation of the long-time kinetics, making use of additional simulation results. We find very good agreement with the prediction of Talbot et al. (i.e., $p=1 / 3$ ) for rectangles with aspect ratios in the range $1 \leqslant \alpha \leqslant 4$. For rectangles with larger aspect ratios, we have not obtained enough data to measure $p$ accurately. However, we believe that the exponent $p$ may also be equal to $1 / 3$ for these cases.

We also carried out a simulation of the RSA of line segments onto a plane $(\alpha=\infty)$, which to our knowledge had not been studied before. In this case, of course, there is no jamming limit. We show that the adsorption rate for rectangles, when properly scaled, approaches that of the line segments as $\alpha$ becomes large.

\section{RESULTS AND DISCUSSION}

The simulation method used in this study has been described previously, ${ }^{9}$ so we shall not repeat it here. We are interested in determining the exponent $p$ in Eq. (1). A simple $\log -\log$ plotting procedure to find $p$ requires that the jamming coverage $\theta_{J}$ be known for the rectangles in question. However, in order to determine $\theta_{J}$ accurately, one must be able to extrapolate the data to $\tau=\infty$ (as no simulation is run completely to jamming). However, this extrapolation can only be carried out if $p$ is known. In order to circumvent this difficulty, one can instead plot $\ln [\theta(2 \tau)-\theta(\tau)]$ against $\ln \tau$, which will yield a straightline plot with slope $-p$ if Eq. (1) is obeyed. By using this method (which is a kind of numerical differentiation of the data), it is not necessary to know $\theta_{J}$ in order to calculate $p$.

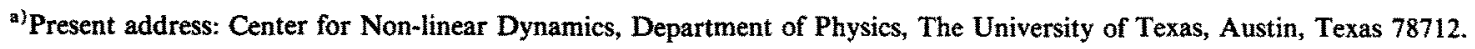




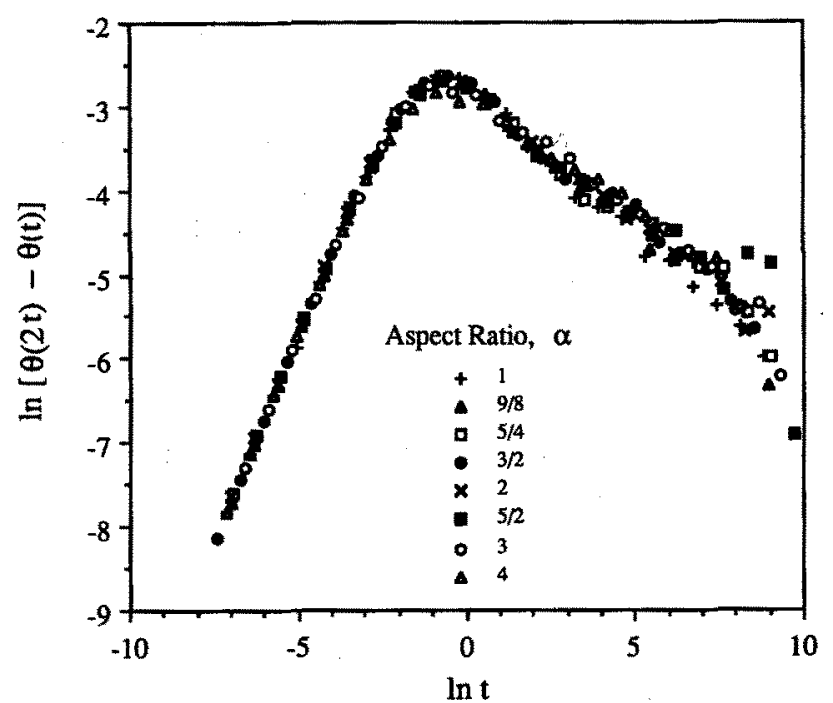

FIG. 1. The plot of $\ln [\theta(2 t)-\theta(t)]$ vs $\ln t$ for various rectangle aspect ratios in the range $1 \leqslant \alpha \leqslant 4$.

In order to compare the simulation results for rectangles of various aspect ratios, a consistent time variable must be defined. We define

$$
t=\left(l w / L^{2}\right) \tau
$$

where $l$ and $w$ are the length and width of the rectangles, respectively, $L$ is the length of the square target surface, and $\tau$ is the total number of attempted adsorption events (both successful and not). That is, a unit of time $t$ passes after $\tau=L^{2} / l w$ adsorption attempts, which equals one adsorption attempt (on average) per rectangular $(l \times w)$ unit surface area. Using this scaled time variable $t$, we plot $\ln [\theta(2 t)-\theta(t)]$ vs $\ln t$ for various rectangle aspect ratios in the range $1 \leqslant \alpha \leqslant 4$ in Fig. 1. There is a remarkable overlap of the curves in this plot, with a peak occurring near $t=1$. Thus, in this scaling of time (where the increment increases in powers of 2), the number of rectangles adsorbed in a given time increment increases until there has been approximately one adsorption attempt per unit surface area. Thereafter, the number of rectangles adsorbed per time increment decreases. In Fig. 1, it is seen that a straight line is indeed obtained at these larger values of time and that the value of the slope agrees with the prediction of Talbot et al., ${ }^{8} p=-1 / 3$ within an error of \pm 0.05 . The overlap of the curves in Fig. 1 suggests that the adsorption kinetics of rectangles is independent of aspect ratio (for $1 \leqslant \alpha \leqslant 4$ ) and it explains the nearly identical values of $\theta_{J}$ obtained for rectangles with aspect ratios in this range.

Rectangles with aspect ratios in the range $8 \leqslant \alpha \leqslant 256$ were also studied and the data is presented in Fig. 2. In these cases, it is difficult to determine the value of $p$ accurately since the asymptotic regime has evidently not been reached. However, the trends in the curves at large times seem consistent with the expected slope of $-1 / 3$. Note

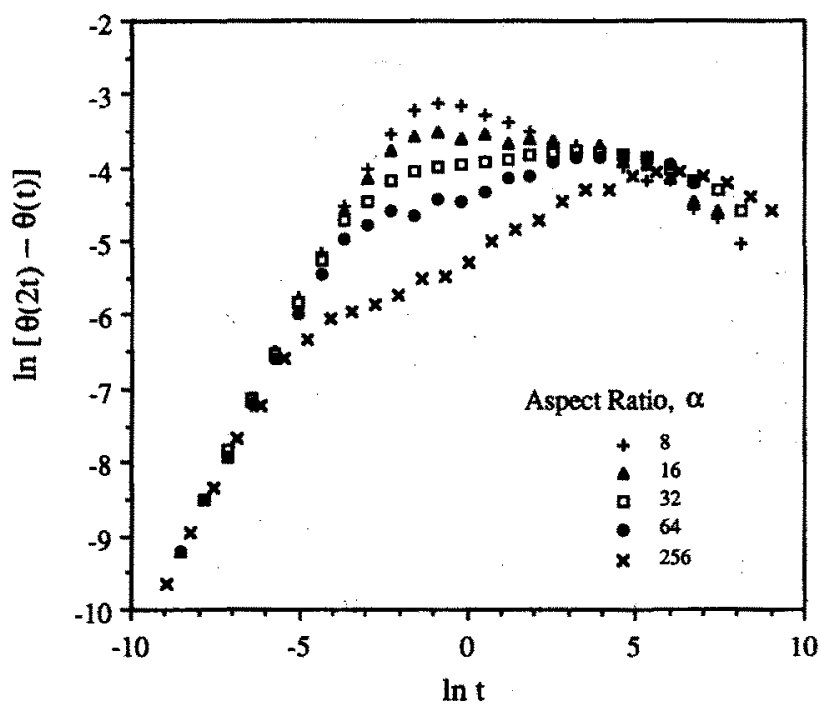

FIG. 2. The plot of $\ln [\theta(2 t)-\theta(t)]$ vs $\ln t$ for various rectangle aspect ratios in the range $8 \leqslant \alpha \leqslant 256$.

that for these cases there is a plateau which occurs before the peak and that these peaks do not line up any longer at $t \approx 1$.

To understand the behavior of rectangles with large $\alpha$, we have also considered the RSA of line segments onto a plane $(\alpha=\infty)$. In this case, there is only one length scale $l$, since $w=0$. Thus, we define a different time scale

$$
t^{\prime}=(l / L)^{2} \tau \text {. }
$$

One unit of time $t^{\prime}$ passes when there has been one adsorption attempt (on average) per unit area, defined as the area of a square with length $l$. Equation (4) allows us to compare the adsorption kinetics of line segments with those of rectangles. For example, Fig. 3 shows a plot of $\ln \left[\theta\left(2 t^{\prime}\right)-\theta\left(t^{\prime}\right)\right]$ vs $\ln t^{\prime}$ for line segments and rectangles with aspect ratios in the range $1 \leqslant \alpha \leqslant 256$. Notice that the

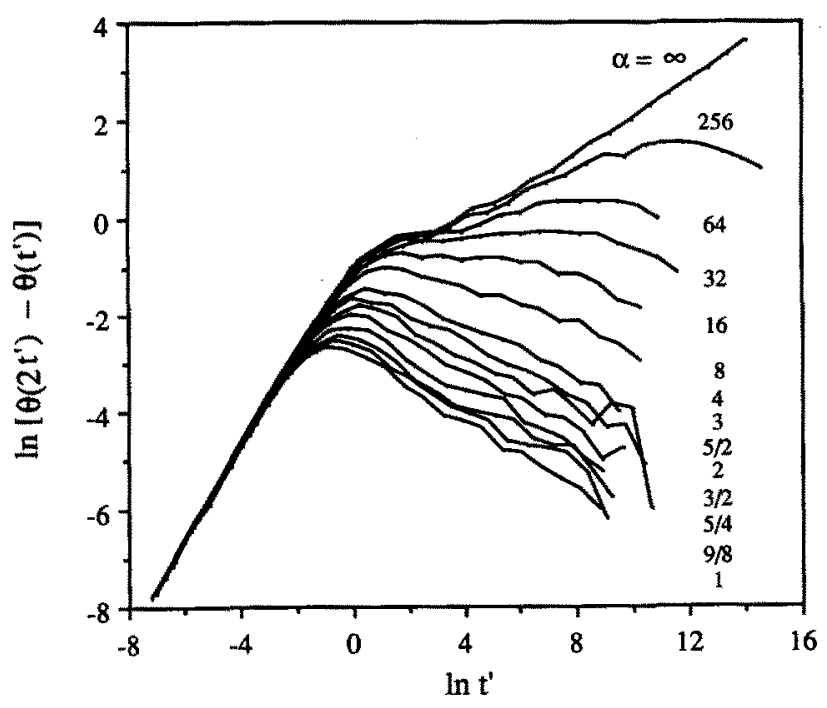

FIG. 3. The plot of $\ln \left[\theta\left(2 t^{\prime}\right)-\theta\left(t^{\prime}\right)\right]$ vs $\ln t^{\prime}$ for line segments and rectangles with aspect ratios in the range $1 \leqslant \alpha \leqslant 256$. 
curves for the rectangles overlap the curve for the line segments at short times and then deviate at successively longer values of $t^{\prime}$ with increasing aspect ratio. At short times, the slopes of the curves in all cases are approximately equal to 1 , indicating that nearly every adsorption attempt is successful. At long times, however, the curve for the line segments never reaches a maximum (since no jamming state exists), but instead appears to increase linearly with a slope of $0.35 \pm 0.05$. The plateau that occurs at intermediate times in Fig. 2 for rectangles and Fig. 3 for line segments and rectangles with large aspect ratios is a result of the transition between these two power-law regimes.

For rectangles with large aspect ratios, the adsorption kinetics are very close to those of the infinitely thin lines for a long period of time. During this time, nearly all rejections of rectangles are due to overlaps of their long axes, in which case, the rectangles act identically to lines. Here, the chance to overlap just the width of the rectangles (without crossing the long axis) is much smaller than the chance to adsorb in an open space. However, when the system approaches the jamming limit, only small spaces are left for adsorption and the chance of adsorbing in an open space now becomes much less than the chance of overlapping on the width of the rectangles, and the kinetics of the rectangles deviate from those of the line segments.
The time at which these restrictions due to the rectangle width become important increases with increasing aspect ratio.

In summary, we find that the approach to the jamming limit for the RSA of rectangles is consistent with the prediction of Talbot $e t$ al. ${ }^{8}$ and that the kinetics of adsorption of rectangles approaches those of line segments as $\alpha$ becomes large.

\section{ACKNOWLEDGMENT}

We acknowledge National Science Foundation Grant No. DMR-8619731 for support of this research.

A. Renyi, Publ. Math. Inst. Hung. Acad. Sci. 3, 109 (1958).

${ }^{2}$ J. K. MacKenzie, J. Chem. Phys. 37, 723 (1962).

${ }^{3}$ B. Widom, J. Chem. Phys. 44, 3888 (1966).

${ }^{4}$ J. J. Gonzalez, P. C. Hemmer, and J. S. Hoye, Chem. Phys. 3, 228 (1974).

${ }^{5}$ J. W. Evans, J. Phys. A: Math. Gen. 23, 2227 (1990)

${ }^{6}$ P. Schaaf and J. Talbot, Phys. Rev. Lett. 62, 175 (1989).

${ }^{7}$ A. Baram and D. Kutasov, J. Phys. A: Math. Gen. 22, L251 (1989)

${ }^{8}$ J. Talbot, G. Tarjus, and P. Schaaf, Phys. Rev. A 40, 4808 (1989).

${ }^{9}$ R. D. Vigil and R. M. Ziff, J. Chem. Phys. 91, 2599 (1989).

${ }^{10}$ J. Feder, J. Theor. Biol. 87, 237 (1980).

${ }^{11}$ Y. Pomeau, J. Phys. A: Math. Gen. 13, L193 (1980).

${ }^{12}$ R. H. Swendsen, Phys. Rev. A 24, 504 (1981).

${ }^{13}$ R. M. Ziff, B. Brosilow, and R. D. Vigil, Phys. Rev. A (submitted). 